\title{
Maximal Minimum Hamming Distance Codes for Embedding SI in a Data-based BSLM Scheme for PAPR Reduction in OFDM
}

\author{
Adnan Haider Yusef Sa'd ${ }^{1,2}$, Hisham Haider Yusef Saad ${ }^{1}$, Aeizaal Azman Abd Wahab ${ }^{2 *}$ \\ ${ }^{1}$ Faculty of Computer and IT, Al-Razi University, Sana'a, Yemen \\ ${ }^{2}$ School of Electrical \& Electronic Engineering, Universiti Sains Malaysia, Penang 14300, Malaysia
}

\begin{abstract}
The bi-orthogonal codes for embedding Side-Information (SI) in data-based blind SLM (BSLM) proposed in Joo et al. (2012) produce better bit error rate (BER) and SI error rate (SIER) performance compared to binary codes. However, the authors do not provide details for code generation; instead, they list some codes with a length of $\boldsymbol{U} / \mathbf{2}$ and a minimum Hamming distance of $\boldsymbol{U} / \mathbf{4}$. The suggested bi-orthogonal code does not work for any value of the maximum iteration number $\boldsymbol{U}$ other than $\boldsymbol{U}=\mathbf{4}[\boldsymbol{U} / \mathbf{4}\rceil$. Therefore, this paper proposes two algorithms for generating $\boldsymbol{U}$ codes for any value of $\boldsymbol{U}$. The proposed methods maintain the normalized minimum Hamming distance between generated codes to $\mathbf{0 . 5}$. However, the second proposed algorithm, which works in the case of $\boldsymbol{U}<\left\lfloor\log _{2} \boldsymbol{U}\right\rfloor$, is also able to only consider codes with a maximum Hamming distance, allowing it to improve SIER performance. Thus, the second proposed algorithm improves SIER performance by up to $1 \mathrm{~dB}$ at $\mathrm{Eb} / \mathrm{No}=3 \mathrm{~dB}$. Furthermore, the proposed algorithms are able to generate a multiple set of $\boldsymbol{U}$ codes that deliver same performance.
\end{abstract}

Keywords: Blind SLM; OFDM; PAPR; Side Information; SLM

\section{Introduction}

Orthogonal frequency division multiplexing (OFDM) is a transmission technique with a high data-transmission rate and can cope with severe channel conditions. Moreover, unlike other techniques that utilize parallelism for increasing transmission speed, the orthogonal nature of OFDM uses bandwidth more efficiently. OFDM has been adopted by many standards for its advantages over other techniques, such as single carrier or frequency division multiplexing (FDM). Examples of well-known standards include DSL, 802.11a, WiFi-Max, and LTE (Han and Lee, 2005; Jiang and Wu, 2008; Rahmatallah and Mohan, 2013).

Thanks to advances in digital signal processing (DSP) technology, OFDM has become more widely applicable and popular. However, OFDM suffers from the major problem of having high signal peaks. The high peaks problem, often called the high peak-to-average power ratio (PAPR) problem, is a result of the summation of multiple subcarrier signals due to the existence of a parallelism concept in transmission. The high peaks of an OFDM signal cannot be amplified linearly using a normal power amplifier (PA); doing so corrupts the signal, as there are high peaks in the non-linear region of PA. Thus, as using normal PA with OFDM introduces in-band and out-band radiation, OFDM requires a very complex PA design

*Corresponding author's email: aeizaal@usm.my, Tel.: +60-124211107

doi: 10.14716/ijtech.v12i2.4350 
to maintain linearity. Mathematically, PAPR is calculated as follows:

$$
\operatorname{PAPR}(x[n])=\frac{\max \left(|x[n]|^{2}\right)}{E\left(|x[n]|^{2}\right)}
$$

where $x[n]$ is the $n$th sample of OFDM time domain signal $x$, and $E$ denotes expectation.

Many methods have been proposed to alleviate the high PAPR problem; each one has advantages and disadvantages. Examples of PAPR reduction techniques found in the literature are clipping (Juwono et al., 2013), partial transmit sequence (PTS) (Müller et al., 1997; Müller and Huber, 1997), selected mapping (SLM) (Müller et al., 1997; Sa'd and Wahab, 2019), tone injection (TI) (Wattanasuwakull and Benjapolakul, 2005), tone reservation (TR) (Park et al., 2003), interleaving (Rahmatallah and Mohan, 2013), and hybrid (Jones et al., 1994; Wang and Chen, 2014; Sa'd et al., 2016; Wahab and Sa'd, 2017; Liang et al., 2019).

SLM is a well-known distortion-less PAPR reduction technique; however, it requires sending side information (SI) for the receiver to be able to retrieve the original signal. To solve this problem, many blind SLM (BSLM) schemes have been proposed in the literature (Jayalath and Tellambura, 2002; Pezeshk and Khalaj, 2002; Baxley and Zhou, 2005; Jayalath and Tellambura, 2005; Han et al., 2006; Chen and Zhou, 2006;Alsusa and Yang, 2008; Han et al., 2008; Joo et al., 2009; Le Goff et al., 2009; Hong et al., 2010; Park et al., 2011; Li et al., 2011; Badran and El-Helw, 2011;Eom et al., 2012; Joo et al., 2012; El-Helw et al., 2012; Hong et al., 2013; Ji and Ren, 2013; Elhelw and Badran, 2015; Ji et al., 2015; Yoon et al., 2018; Goel and Sidhu, 2020). BSLM, in general, embeds SI in an OFDM signal by utilizing noise margins instead of dedicated subcarriers to eliminate data rate waste due to SI. While there are different types of BSLM, the data-based BSLM proposed in Joo et al. (2012) can maintain a similar PAPR and an almost similar BER as conventional SLM without manipulating or imposing restrictions on channel estimation methods, providing that the number of subcarriers of OFDM is not very small. SI is embedded onto the phase of data subcarriers by making a unique and distinguished phase disparity between all the possible iterations of data manipulation, and this is done over all data subcarriers. To improve SI error rate (SIER) performance, phase disparity forms are constructed using a biorthogonal vector with a code length of $L=U / 2$. However, the work in Joo et al. (2012) lacks the generalization of constructing biorthogonal codes for any $U$ value. Therefore, in this paper, we propose a systematic way of generating $U$ SI embedding-codes that fulfill the general and important condition of having a normalized minimum Hamming distance of at least 0.5.

This paper is organized as follows. Section 2 explains the data-based BSLM in Joo et al. (2012). Section 3 discusses SI embedding-code generation and explains the proposed maximal Hamming distance code generation method. Finally, a conclusion is drawn in Section 4 .

\section{Data-based BSLM}

Let us assume the OFDM modulated input sequence $X=\left[X_{1}, X_{2}, \ldots, X_{N}\right], X \in \mathcal{Q}$, where $\mathcal{Q}$ denotes the modulation's constellation points; to reduce the PAPR value of the OFDM signal, BSLM iterates $U$ different phase rotation operations on the input signal and selects the one with the lowest PAPR for transmission. This is done by multiplying the sequence $X$ with different phase rotation sequences as follows:

$$
S^{(u)}=X \cdot P^{(u)}
$$

where $u \in\{0,1, \ldots, U-1\}$ denotes the iteration index, $P^{(u)} \in\{ \pm 1, \pm j\}$ denotes the $u$ th phase rotation sequence, and $S^{(u)} \in \mathcal{Q}$. Then, unlike SLM, BSLM embeds SI into the phase 
sequence of the OFDM data sequence so that the form of phase disparity in $S^{(u)}$ is unique and corresponds to the value of $u$. The phase disparity is formed by moving specific and particular subcarriers' symbols of the sequence $S^{(u)}$ from one constellation diagram $\mathcal{Q}$ into another one $\mathcal{Q}_{\theta}$, rotated by $\theta$, which is given by

$$
\mathcal{Q}_{\theta}=\mathcal{Q} \cdot e^{j \theta}
$$

where $\mathcal{Q}_{\theta}$ is the rotated version of the original constellation diagram $\mathcal{Q}$, and $\theta$ denotes the rotation phase. Through an exhaustive search, the optimum rotation phase $\theta$ that delivers the maximum Euclidean distance between $\mathcal{Q}$ and $\mathcal{Q}_{\theta}$ is found to be $\pi / 4$. Therefore, Equation 3 can be rewritten as

$$
\mathcal{Q}_{\frac{\pi}{4}}=\mathcal{Q} \cdot e^{j \frac{\pi}{4}}
$$

The impact of phase rotation on constellation points is depicted in Figure 1 for both QPSK and 16-QAM modulations to illustrate the minimum distance between the points of both $\mathcal{Q}$ and $\mathcal{Q}_{\pi / 4}$ under a no-noise condition. To combat the reduced noise margin per symbol, phase disparity is implemented over all OFDM data symbols rather than just some of the symbols. The phase disparity between all $U$ possible $S^{(u)}$ symbols is controlled using the phase disparity vectors $\Psi$ as follows:

$$
\begin{aligned}
\mathbf{S}^{(u)} & =S^{(u)} \cdot \Psi^{(u)} \\
& =X \cdot P^{(u)} \cdot \Psi^{(u)}
\end{aligned}
$$

where $S^{(u)}=X . P^{(u)} \in \mathcal{Q}, \mathbf{S}^{(u)} \in\left\{\mathcal{Q}, Q_{\frac{\pi}{4}}\right\}$, and $\Psi^{(u)} \in\left\{e^{j 0}=1, e^{j \pi / 4}\right\}$. It is clear that the constellation diagram of the $n$th subcarrier's symbol of the $u$ th iteration $\mathbf{S}_{n}^{(u)}$ is decided by $\Psi_{n}^{(u)}$ as follows:

$$
\mathbf{S}_{n}^{(u)} \in\left\{\begin{array}{cc}
\mathcal{Q}, & \Psi_{n}^{(u)}=1 \\
\mathcal{Q}_{\frac{\pi}{4}}, & \Psi_{n}^{(u)}=e^{j \frac{\pi}{4}}
\end{array}\right.
$$
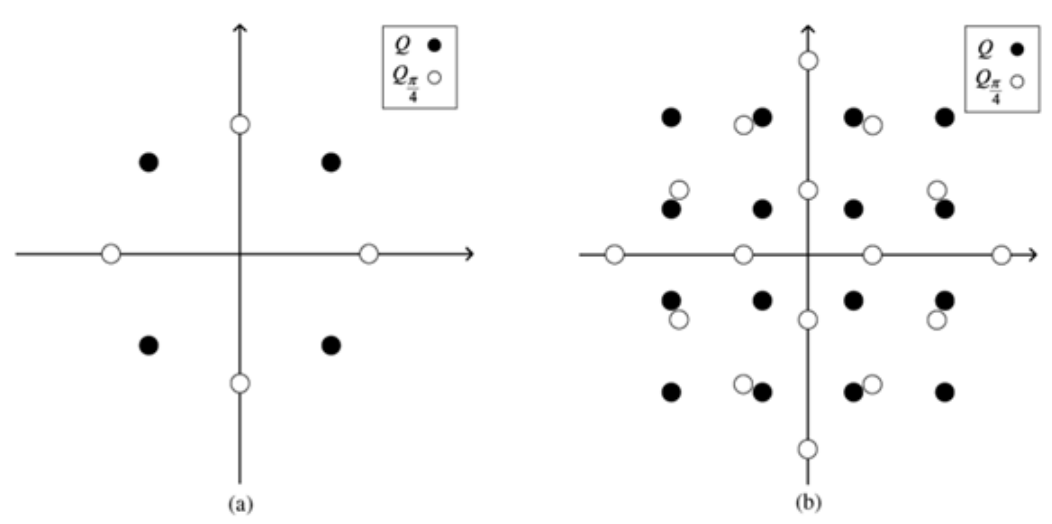

Figure 1 Signal constellations $\mathcal{Q}$ and $\mathcal{Q}_{\frac{\pi}{4}}$ : (a) QPSK; and (b) 16-QAM (Joo et al., 2012)

In general, the phase disparity control vector $\Psi_{n}^{(u)} \in\left\{e^{j \frac{\pi}{4} \rho}\right\}$ where $\rho \in\{0,1\}$. Therefore, the vector $\Psi^{(u)}$ can be constructed using any binary code with length $L$. For instance, assume $C=\left[C^{(0)}, C^{(1)}, \ldots, C^{(U-1)}\right]$ is the binary code of the iteration indices $u$ and the length of $C^{(u)}=\left[C_{1}^{(u)}, C_{2}^{(u)}, \ldots, C_{L}^{(u)}\right]$ is $L \geq\lceil\log 2(U)\rceil$. Then, the phase disparity control vector is given by

$$
\Psi^{(u)}=\left\{\Psi_{n}^{(u)}=e^{j \phi C_{\ell}^{(u)}} \mid n=1,2, \ldots, N\right\}, \quad \ell=\left\lceil\frac{n}{N / L}\right\rceil
$$


where $\ell=[1,2, \ldots, L]$ denotes the sub-block's index, and $C_{\ell}^{(u)}$ represent the corresponding $\ell$ th bit of the SI embedding codeword $C^{(u)} \in C$ for the iteration index $u$.

It can be seen that Equation 7 divides the disparity control vectors $\Psi$ into sub-blocks of two values only $\left(e^{j \theta \rho}, \rho \in\{0,1\}\right)$, where the elements in the same sub-block are equal. As a result, the phase disparity control vectors also divide OFDM sub-symbols in Equation 5 into sub-blocks but of two constellation diagrams (i.e. $\mathbf{S}^{(u)} \in\left\{\mathcal{Q}, \mathcal{Q}_{\theta}\right\}$ ). Table 1 and Table 2 show an example of $\Psi$ construction and an example of its influence on OFDM symbols in Equation 5, respectively, for $U=2$ and $N=256$.

Finally, only one out of $U \mathbf{S}^{(u)}$ signals will be transmitted, and the index of the selected signal for transmission is found as follows:

$$
\check{u}=\arg \min _{0 \leq u<U} \max \left(\operatorname{IFFT}\left(S^{(u)}\right)\right)
$$

Table 1 Phase disparity control vectors for $U=4$ and $N=256$

\begin{tabular}{ccccc}
\hline & & $C^{(u)}$ & \multicolumn{2}{c}{$\Psi^{(u)}$ is divided into $L=2$ sub-blocks } \\
\cline { 2 - 5 } & $C_{1}^{(u)}$ & $C_{2}^{(u)}$ & $1,2,3, \ldots .128$ & $129,130, \ldots .256$ \\
\hline 0 & 0 & 0 & $1,1,1, \ldots, 1$ & $1,1,1, \ldots, 1$ \\
1 & 0 & 1 & $1,1,1, \ldots, 1$ & $e^{j \theta}, e^{j \theta}, \ldots, e^{j \theta}$ \\
2 & 1 & 0 & $e^{j \theta}, e^{j \theta}, \ldots, e^{j \theta}$ & $1,1,1, \ldots ., 1$ \\
3 & 1 & 1 & $e^{j \theta}, e^{j \theta}, \ldots, e^{j \theta}$ & $e^{j \theta}, e^{j \theta}, \ldots, e^{j \theta}$ \\
\hline
\end{tabular}

Table 2 Impact of phase disparity control vectors on OFDM symbols for $U=4$ and $N=256$

\begin{tabular}{|c|c|c|c|c|}
\hline \multirow{2}{*}{$u$} & \multicolumn{2}{|c|}{$C^{(u)}$} & \multicolumn{2}{|c|}{$\begin{array}{l}\mathbf{S}^{(u)} \text { is divided into } L=2 \text { sub-blocks of } \\
\text { values members of } \mathcal{Q} \text { or } \mathcal{Q}_{\theta}\end{array}$} \\
\hline & $C_{1}^{(u)}$ & $C_{2}^{(u)}$ & $1,2,3, \ldots .128$ & $129,130, \ldots .256$ \\
\hline 0 & 0 & 0 & $\mathcal{Q}, \mathcal{Q}, \mathcal{Q}, \ldots, \mathcal{Q}$ & $Q, Q, Q, \ldots, \mathcal{Q}$ \\
\hline 1 & 0 & 1 & $\mathcal{Q}, \mathcal{Q}, \mathcal{Q}, \ldots, \mathcal{Q}$ & $\mathcal{Q}_{\theta}, \mathcal{Q}_{\theta}, \ldots, \mathcal{Q}_{\theta}$ \\
\hline 2 & 1 & 0 & $\mathcal{Q}_{\theta}, \mathcal{Q}_{\theta}, \ldots, \mathcal{Q}_{\theta}$ & $\mathcal{Q}, \mathcal{Q}, \mathcal{Q}, \ldots, \mathcal{Q}$ \\
\hline 3 & 1 & 1 & $\mathcal{Q}_{\theta}, \mathcal{Q}_{\theta}, \ldots, \mathcal{Q}_{\theta}$ & $\mathcal{Q}_{\theta}, \mathcal{Q}_{\theta}, \ldots, \mathcal{Q}_{\theta}$ \\
\hline
\end{tabular}

\subsection{Decoding Algorithm}

At the receiver, BSLM adopts maximum likelihood (ML) decoding to estimate the SI, as shown in Figure 2. Mathematically, the received signal is denoted as $R$, and it is given by

$$
R=X P^{(u)} \Psi^{(\mathrm{u})} H+\eta
$$

where $\eta$ denotes the white Gaussian noise and $H$ denotes the channel coefficients. Then, the BSLM receiver calculates the minimum distance between every received symbol $R_{n} \in R$ to both constellation diagrams $\mathcal{Q}$ and $\mathcal{Q}_{\theta}$, and then calculates the corresponding total minimum distance per sub-block $\ell=[1,2, \ldots, L]$. 


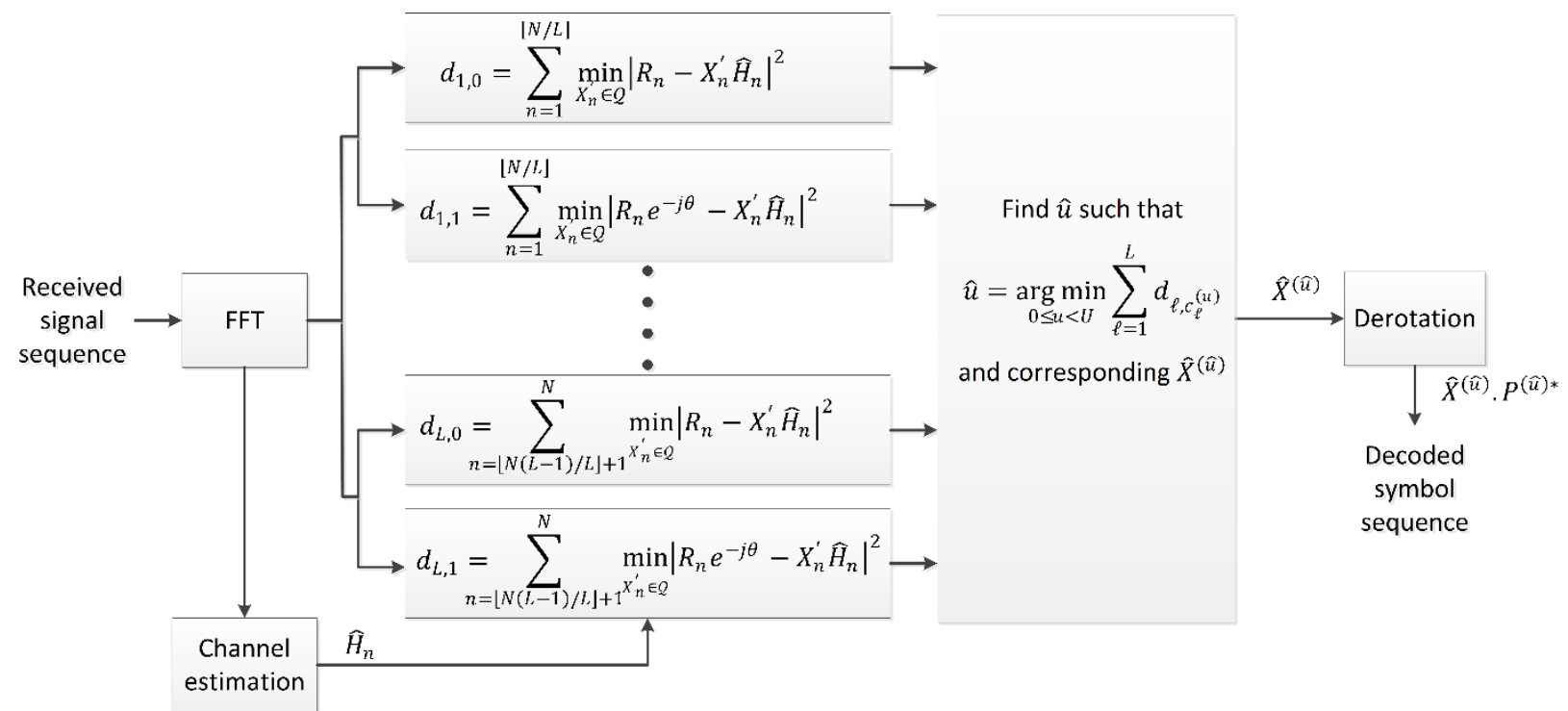

Figure 2 BSLM receiver block diagram

Mathematically, the total minimum distance per sub-block and constellation diagram can be calculated as follows:

$$
d_{\ell, p}=\sum_{n=\left\lfloor\frac{N(\ell-1)}{L}\right\rfloor+1}^{\left\lfloor\frac{N \ell}{L}\right\rfloor} \min _{X_{n}^{\prime} \in \mathcal{Q}}\left|R_{n} e^{-j \theta p}-X_{n}^{\prime} \widehat{H}_{n}\right|^{2}
$$

where $p \in\{0,1\}, d_{\ell, p}$ denotes the metric or the total minimum distance per sub-block $\ell$ and the constellation diagram $\mathcal{Q} e^{j \theta p}$, and $\widehat{H}_{n}$ represents the estimated channel response. Finally, the iteration index is estimated as follows:

$$
\hat{u}=\min _{0 \leq u<U} \sum_{\ell=1}^{L} d_{\ell, c_{\ell}^{(u)}}
$$

\section{BSLM SI Embedding Codes}

The simplest SI embedding codes are the straight binary representation of the iteration indices. However, binary codes with code length $L=\lceil\log 2(U)\rceil$ have poor SIER compared to other codes.

Table 3 Biorthogonal vectors for embedding SI for $\boldsymbol{U}=\mathbf{4}$ and $\boldsymbol{U}=\mathbf{8}$, as proposed in Joo et al. (2012)

\begin{tabular}{ccccccc}
\hline \multirow{2}{*}{$\begin{array}{c}\text { Iteration } \\
\text { Index } u\end{array}$} & \multicolumn{5}{c}{ SI Embedding Codes } \\
\cline { 2 - 7 } & \multicolumn{2}{c}{$U=4$} & \multicolumn{4}{c}{$U=8$} \\
\hline 0 & 0 & 0 & 0 & 0 & 0 & 0 \\
1 & 0 & 1 & 0 & 0 & 1 & 1 \\
2 & 1 & 0 & 0 & 1 & 1 & 0 \\
3 & 1 & 1 & 0 & 1 & 0 & 1 \\
4 & & & 1 & 1 & 0 & 0 \\
5 & & & 1 & 0 & 1 & 0 \\
6 & & & 1 & 0 & 0 & 1 \\
7 & & & 1 & 1 & 1 & 1 \\
\hline
\end{tabular}


Therefore, Joo et al. (2012) suggest constructing SI embedding codes using biorthogonal vectors with the codeword length $L=U / 2$. However, the work in Joo et al. (2012) does not clearly explain code construction; instead, the authors provide an example for $U=4$ and 8 , as shown in Table 3. Thus, the following section of this paper proposes generalized algorithms for constructing $U$ codewords that possess the main feature of biorthogonal vectors (i.e. the minimum Hamming distance of $L / 2$ ). The proposed algorithms generate $U$ codes for any value of $U$ (i.e. $U$ doesn't necessarily need to be multiple of 2 ).

\subsection{Proposed Maximal Hamming Distance Codes}

In the proposed algorithm for generating maximal Hamming distance codes, the generated codes need to fulfill one key feature: the normalized minimum Hamming distance $d_{\text {min }} / L$ between all generated codewords should equal 0.5 , where $d_{\text {min }}$ represents the minimum Hamming distance between all generated codewords. The generation of such code is possible for codeword lengths (the number of sub-blocks) of $L=$ $(U / 2) \in \mathbb{N}$. However, this is not true for every value of $U$. Therefore, generally speaking, for any value of $U$, codeword length $L$ can be obtained and determined as follows:

$$
L=2^{\lceil\log 2(U)\rceil+k} / 2, \quad k \in \mathbb{N}
$$

and, for simplicity, we can set $k=0$ from now on. Therefore, Equation 12 changes to

$$
L=2^{\lceil\log 2(U)\rceil} / 2
$$

Then, a generalized method for constructing a binary code with $L$ given in Equation 13 can be employed by conducting a simple computer search using the following algorithm:

Algorithm 1: SI embedding code generation algorithm.

1- Set $L=2^{\lceil\log 2(U)\rceil} / 2, X=\left[0,1, \ldots, 2^{L}-1\right]$, and $u=0$.

2- $\operatorname{Set} C^{(u)}=X_{1}$

3- Recalculate $X=\left\{X_{n} \mid w\left(X_{1} \oplus X_{n}\right) \geq L / 2\right\}$ for all $X_{n}$ values in $X(w($.$) denotes the$ weight, and $\oplus$ denotes XOR operation). Then, increase $u$ by 1 .

4- If $u<U$, go to Step 2. Otherwise, Return.

Table 4 SI embedding codes generated by Algorithm 1 for $\boldsymbol{U}=\mathbf{8}$

\begin{tabular}{ccc}
\hline$u$ & \multicolumn{2}{c}{ SI Embedding Codes $\Psi$} \\
\cline { 2 - 3 } & Option 1 & Option 2 \\
\hline 0 & 0 & 1 \\
1 & 3 & 2 \\
2 & 5 & 4 \\
3 & 6 & 7 \\
4 & 9 & 8 \\
5 & 10 & 11 \\
6 & 12 & 13 \\
7 & 15 & 14 \\
\hline
\end{tabular}


It is worth noting that there are at least $2^{L-[\log 2(U)]}$ vectors of $U$ elements with $d_{\min } / L=0.5$. For instance, for $U=4$ and $L=2$, there is only one vector with $d_{\min } / L=$ 0.5 . However, there are two vectors that fulfill the minimum distance requirement for $U=$ 8 and $L=3$, as shown in Table 4, and the number increases as the number of iterations $U$ increases or, more precisely, as $\lceil\log 2(U)\rceil$ increases.

Table 4 shows that for $U=8$, there are $2^{L-\lceil\log 2(U)\rceil}=2$ SI embedding codes options, where the first one (option 1 ) is similar to, but in a different order than, the codes proposed in Joo et al. (2012) for $U=8$, as shown in Table 3. It is worth noting that alternative codes (e.g. for $U=8$, Option 2 in Table 4) can be found by applying Algorithm 1 to the vector $X$, excluding all previously found code, given as follows:

$$
X=\left\{X_{n} \mid\left\{0,1, \ldots, 2^{L}-1\right\} \ni X_{n} \notin \mathbf{C}\right\}
$$

where $\mathbf{C}$ denotes all previously found codes using the algorithm. Furthermore, in the case of $U<2 L$, Algorithm 1 should be modified to ensure that, for an even value of $U$, every $C^{(u)} \in C$ and its invert $\overline{C^{(u)}}$ belongs to the set $C$ composed of $U$ codes. This is important because, although the minimum Hamming distance for $C$ is $L / 2$, the Hamming distance between $\left\{C^{(u)}, \overline{C^{(u)}}\right\} \in C$ is $L$. Algorithm 2 illustrates how to construct $U<2 L$ SI embedding codes with $d_{\min }=L / 2$.

Algorithm 2: SI embedding code generation algorithm for $U<2 L$

1- Set $L=2^{[\log 2(U)]} / 2, X=\left[0,1, \ldots, 2^{L-1}-1\right]$, and $u=0$.

2- Set $C^{(u)}=X_{1}$. Then increase $u$ by 1 .

3- If $u<U$, then set $C^{(u)}=\overline{X_{1}}=2^{L}-1-X_{1}$. Otherwise, Return.

4- Recalculate $X=\left\{X_{n} \mid w\left(X_{1} \oplus X_{n}\right) \geq L / 2\right\}$ for all $X_{n}$ values in $X(w($.$) denotes the$ weight, and $\oplus$ denotes XOR operation). Then increase $u$ by 1 .

5- If $u<U$, then go to Step 2. Otherwise, Return.

Although both Algorithm 1 and Algorithm 2 are capable of generating $U$ codes with $d_{\text {min }}=L / 2$ for the case of $U<2 L$, Algorithm 2 also ensures that almost all codewords in $C$ have a maximum Hamming distance of $L$. For instance, in the case of $U=5<2 L$ and $L=4$, the SI embedding code generated by Algorithm 1 is $C=[0,3,5,6,9]$; however, in Algorithm 2 , it is $C=[0,15,3,12,5]$. We can see that both generated codes have $d_{\text {min }}=L / 2$; however, the codes generated by Algorithm $1(C=[0,3,5,6,9])$ have a maximum Hamming distance $d_{\text {max }}=L$ between $\{6,9\}$ only, while the codes generated by Algorithm 2 have $d_{\text {max }}=L$ between both $\{0,15\}$ and $\{3,12\}$. For this reason, Algorithm 2 produces better SIER performance, as shown in Figure 3. 


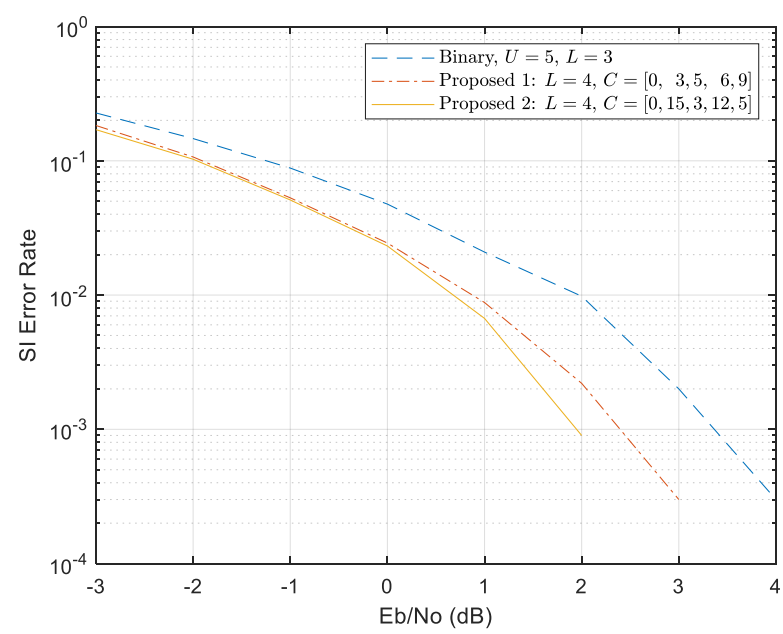

Figure 3 SIER performance evaluation for data-based BSLM with 16-QAM modulation; $N=256$, $U=5$, and three different SI embedding codes (simple binary representation of iteration indices (i.e. $[0,1,2,3,4]$ ) versus the two different maximal Hamming distance codes generated by Algorithm 1 and Algorithm 2 proposed in this article) in the AWGN channel

\section{Conclusions}

Two new algorithms were proposed for generating maximal Hamming distance codes of $d_{\min }=L / 2$ for embedding SI. The proposed algorithms generalize the construction of SI embedding codes for any value of $U$. The codes produced using the algorithms proposed here and the biorthogonal codes used in Joo et al. (2012) have similar SIER performance for $U=2 L$, since they all have a normalized minimum distance of $d_{\min } / L=0.5$. However, for $U<2 L$, Algorithm 2 proposed here produces better SIER performance.

\section{Acknowledgements}

This research was supported partially by Research University Grant, Universiti Sains Malaysia (1001/PELECT/8014160).

\section{References}

Alsusa, E., Yang, L., 2008. Redundancy-Free and BER-Maintained Selective Mapping with Partial Phase-Randomising Sequences for Peak-to-Average Power Ratio Reduction in OFDM Systems. IET Communications, Volume 2, pp. 66-74

Badran, E.F., El-Helw, A.M., 2011. A Novel Semi-Blind Selected Mapping Technique for PAPR Reduction in OFDM. IEEE Signal Processing Letters, Volume 18(9), pp. 493-496

Baxley, R.J., Zhou, G.T., 2005. MAP Metric for Blind Phase Sequence Detection in Selected Mapping. IEEE Transactions on Broadcasting, Volume 51(4), pp. 565-570

Chen, N., Zhou, G.T., 2006. Peak-to-Average Power Ratio Reduction in OFDM with Blind Selected Pilot Tone Modulation. IEEE Transactions on Wireless Communications, Volume 5(8), pp. 2210-2216

El-Helw, A.M., Badran, E.F., Al-Kafrawy, H.Y., 2012. A New Sequence for Embedding Side Information in SLM for PAPR Reduction in OFDM. In: Japan-Egypt Conference on Electronics, Communications and Computers (JEC-ECC), 2012, pp. 53-56

Elhelw, A.M., Badran, E.F., 2015. Semi-Blind Error Resilient SLM for PAPR Reduction in OFDM using Spread Spectrum Codes. PloS ONE, Volume 10(5), pp. e0127639 
Eom, S.-S., Nam, H., Ko, Y.-C., 2012. Low-Complexity PAPR Reduction Scheme Without Side Information for OFDM Systems. IEEE Transactions on Signal Processing, Volume 60(7), pp. 3657-3669

Goel, A., Sidhu, K., 2020. PAPR Reduction in MIMO-OFDM System using SLM Without SI. Journal of Optical Communications, Volume 41(3), pp. 325-330

Han, S.H., Cioffi, J.M., Lee, J.H., 2006. Tone Injection with Hexagonal Constellation for Peakto-Average Power Ratio Reduction in OFDM. IEEE Communications Letters, Volume 10(9), pp. 646-648

Han, S.H., Cioffi, J.M., Lee, J.H., 2008. On the Use of Hexagonal Constellation for Peak-toAverage Power Ratio Reduction of an ODFM Signal. IEEE Transactions on Wireless Communications, Volume 7(3), pp. 781-786

Han, S.H., Lee, J.H., 2005. An Overview of Peak-to-Average Power Ratio Reduction Techniques for Multicarrier Transmission. Wireless Communications, IEEE, Volume 12(2), pp. 56-65

Hong, E., Kim, H., Yang, K., Har, D., 2013. Pilot-Aided Side Information Detection in SLMBased OFDM Systems. IEEE Transactions on Wireless Communications, Volume 12(7), pp. 3140-3147

Hong, E., Mi, S., Har, D., 2010. SLM-Based OFDM System Without Side Information for Data Recovery. Electronics Letters, Volume 46(3), 271-272

Jayalath, A., Tellambura, C., 2002. A Blind SLM Receiver for PAR-Reduced OFDM. In: Proceedings of the Vehicular Technology Conference, 2002, pp. 219-222

Jayalath, A.D.S., Tellambura, C., 2005. SLM and PTS Peak-Power Reduction of OFDM Signals Without Side Information. IEEE Transactions on Wireless Communications, Volume 4(5), 2006-2013

Ji, J., Ren, G., 2013. A New Modified SLM Scheme for Wireless OFDM Systems Without Side Information. IEEE Signal Processing Letters, Volume 20(11), pp. 1090-1093

Ji, J., Ren, G., Zhang, H., 2015. A Semi-Blind SLM Scheme for PAPR Reduction in OFDM Systems with Low-Complexity Transceiver. IEEE Transactions on Vehicular Technology, Volume 64(6), pp. 2698-2703

Jiang, T., Wu, Y., 2008. An Overview: Peak-to-Average Power Ratio Reduction Techniques for OFDM Signals. IEEE transactions on Broadcasting, Volume 54(2), p. 257-268

Jones, A.E., Wilkinson, T.A., Barton, S., 1994. Block Coding Scheme for Reduction of Peak to Mean Envelope Power Ratio of Multicarrier Transmission Schemes. Electronics letters, Volume 30(25), pp. 2098-2099

Joo, H.-S., Heo, S.-J., Jeon, H.-B., No, J.-S., Shin, D.-J., 2009. A New Blind SLM Scheme with Low Complexity of OFDM Signals. In: Vehicular Technology Conference Fall 2009, pp. 1-5

Joo, H.-S., Heo, S.-J., Jeon, H.-B., No, J.-S., Shin, D.-J., 2012. A New Blind SLM Scheme with Low Decoding Complexity for OFDM Systems. IEEE transactions on broadcasting, Volume 58(4), pp. 669-676

Juwono, F.H., Triprasetyo, Y., Gunawan, D., 2013. Exploiting LDPC Codes for Improving the Performance of Clipped-OFDM System. International Journal of Technology, Volume 4(1), pp. 93-99

Le Goff, S.Y., Al-Samahi, S.S., Khoo, B.K., Tsimenidis, C.C., Sharif, B.S., 2009. Selected Mapping Without Side Information for PAPR Reduction in OFDM. In: IEEE Transactions on Wireless Communications, Volume 8(7), pp. 3320-3325

Li, C., Jiang, T., Zhou, Y., Li, H., 2011. A Novel Constellation Reshaping Method for PAPR Reduction of OFDM Signals. IEEE Transactions on Signal Processing, Volume 59(6), pp. 2710-2719 
Liang, L.Y., Wahab, A.A.A., Alhady, S.S.N., Othman, W.A.F.W., 2019. Discrete Wavelet Transform with Discrete Cosine Transform (DCT) for Peak-to-Average Power Ratio (PAPR) Reduction in OFDM System. International Journal of Advanced Trends in Computer Science and Engineering, Volume 8(5), pp. 2260-2264

Müller, S.H., Bäuml, R.W., Fischer, R.F., Huber, J.B., 1997. OFDM with Reduced Peak-toAverage Power Ratio by Multiple Signal Representation. Annales des télécommunications. Volume 52, pp. 58-67

Muller, S.H., Huber, J.B., 1997. A Novel Peak Power Reduction Scheme for OFDM. In: Personal, Indoor and Mobile Radio Communications, 1997, pp. 1090-1094

Müller, S.H., Huber, J.B., 1997. OFDM with Reduced Peak-to-Average Power Ratio by Optimum Combination of Partial Transmit Sequences. Electronics letters, Volume 33, pp. 368-369

Park, J., Hong, E., Har, D., 2011. Low Complexity Data Decoding for SLM-Based OFDM Systems Without Side Information. IEEE Communications Letters, Volume 15(6), pp. 611-613

Park, S.-E., Yun, S., Kim, J.Y., Park, D.S., Joo, P., 2003. Tone Reservation Method for PAPR Reduction Scheme. In: Project IEEE, p. 802

Pezeshk, A., Khalaj, B.H., 2002. Extended Hexagonal Constellations as a Means of Multicarrier PAPR Reduction. In: Eurasian Conference on Information and Communication Technology, 2002, pp. 926-936

Rahmatallah, Y., Mohan, S., 2013. Peak-to-Average Power Ratio Reduction in OFDM Systems: A Survey and Taxonomy. Communications Surveys \& Tutorials, IEEE, Volume 15(4), pp. 1567-1592

Sa'd, A.H.Y., Wahab, A.A.A., 2019. Novel Technique for PAPR and Average Power Reduction in OFDM Scheme. International Journal of Advanced Trends in Computer Science and Engineering, Volume 8(6), pp. 3632-3634

Sa'd, A.H.Y., Wahab, A.A.A., Sa'ad, H.H.Y., 2016. A New Precoded Partial Transit Sequence Method using the Discrete Cosine Transform for Peak-to-Average Power Ratio Reduction in an Orthogonal Frequency Division Multiplexing System. SCIENCEASIA, Volume 42(6), pp. 423-426

Wahab, A., Sa'd, A.H.Y., 2017. An Improved IDCT-OFDM with Partial Transmit Sequence. In: $9^{\text {th }}$ International Conference on Robotic, Vision, Signal Processing and Power Applications, Springer, 2017, pp. 535-546

Wang, Z., Chen, S., 2014. Reduction PAPR of OFDM Signals by Combining Grouped DCT Precoding with PTS. Journal of Signal and Information Processing, Volume 5(4), pp. 135-142

Wattanasuwakull, T., Benjapolakul, W., 2005. PAPR Reduction for OFDM Transmission by using a Method of Tone Reservation and Tone Injection. In: Information, Communications and Signal Processing, 2005 Fifth International Conference on, IEEE, pp. 273-277

Yoon, E., Hwang, D., Jang, C., Kim, J., Yun, U., 2018. Blind Selected Mapping with Side Information Estimation based on the Received Pilot Signal. In: Wireless Communications and Mobile Computing, 2018 